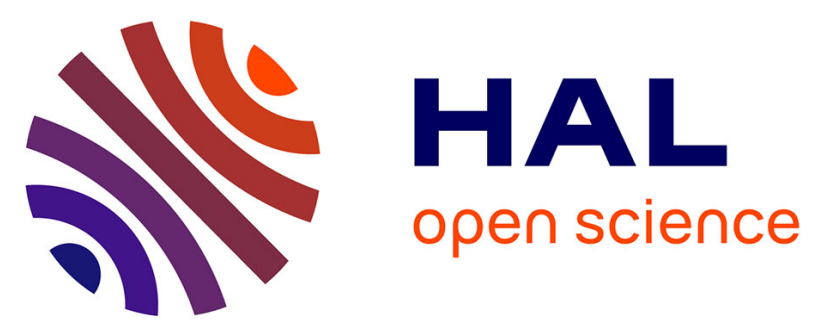

\title{
How an organogelator can gelate water: gelation transfer from oil to water induced by a nanoemulsion
}

Vivien Nouri, Marigilson Pontes de Siqueira Moura, Bruno Payré, Olivier de Almeida, Christophe Dejugnat, Sophie Franceschi-Messant, Emile Perez

\section{- To cite this version:}

Vivien Nouri, Marigilson Pontes de Siqueira Moura, Bruno Payré, Olivier de Almeida, Christophe Dejugnat, et al.. How an organogelator can gelate water: gelation transfer from oil to water induced by a nanoemulsion. Soft Matter, 2020, 16, pp.2371-2378. 10.1039/d0sm00128g • hal-02484264

\author{
HAL Id: hal-02484264 \\ https://hal.science/hal-02484264
}

Submitted on 31 Mar 2020

HAL is a multi-disciplinary open access archive for the deposit and dissemination of scientific research documents, whether they are published or not. The documents may come from teaching and research institutions in France or abroad, or from public or private research centers.
L'archive ouverte pluridisciplinaire HAL, est destinée au dépôt et à la diffusion de documents scientifiques de niveau recherche, publiés ou non, émanant des établissements d'enseignement et de recherche français ou étrangers, des laboratoires publics ou privés. 


\title{
How an organogelator can gelate water: gelation transfer from oil to water induced by a nanoemulsion $\dagger$
}

\author{
Vivien Nouri, ${ }^{a}$ Marigilson Pontes De Siqueira Moura, ${ }^{\mathrm{b}}$ Bruno Payre, ${ }^{\mathrm{c}}$ \\ Olivier De Almeida, ${ }^{d}$ Christophe Déjugnat, (D) ${ }^{a}$ Sophie Franceschi ${ }^{a}$ and \\ Emile Perez (D) *a
}

\begin{abstract}
A hydrogel can be formed by an organogelator in the presence of a nanoemulsion. It is expected that this is due to a gelation transfer from oil to water. The system started with an oil-in-water nanoemulsion prepared according to a phase inversion temperature (PIT) process. Into this nanoemulsion consisting of Kolliphor ${ }^{\circledR}$ RH40 and Brij ${ }^{\circledR}$ L4 as surfactants, and Miglyo ${ }^{\circledR} 812$ as oil and water, we introduced the organogelator 12-hydroxyoctadecanoic acid (12-HOA) in the oil phase. After cooling at room temperature, a slow reversible gelation of the water phase occurred with persistence of the nanoemulsion. This thermally reversible system was investigated using various techniques (rheology, turbidimetry, optical and electron microscopies, scattering techniques). Successive stages appeared during the cooling process after the nanoemulsion formation, corresponding to the migration and self-assembly of the organogelator from the oil nanodroplets to the water phase. According to our measurements and the known self-assembly of 12-HOA, a mechanism explaining the formation of the gelled nanoemulsion is proposed.
\end{abstract}

\section{Introduction}

For several years, we have been developing original aqueous dispersions of organogel particles for drug delivery applications. ${ }^{1-4}$ Such dispersions are prepared by hot emulsification and cooling of an organogel/oil-in-water (o/w) emulsion. Organogels are organic solvents or oils that have been gelled using an organogelator. Most of the organogelators are low molecular weight compounds which, at low concentrations, promote non-covalent self-assembly of structures, generally fibrous, to form a three-dimensional network in solution. ${ }^{5-9}$ Among the low molecular-mass organogelators (LMOGs), we used 12-hydroxyoctadecanoic acid (12-HOA) because it is a biocompatible commercial gelator able to gellify a large variety of organic solvents and oils. ${ }^{10-15}$ So far, we have mostly prepared dispersions for oral drug delivery ${ }^{2,4}$ and we wanted to explore the topical route. Topical delivery systems offer several advantages due to their localized action and the

\footnotetext{
${ }^{a}$ Laboratoire des IMRCP, Université de Toulouse, CNRS UMR 5623, Université Toulouse III - Paul Sabatier, France.E-mail: perez@chimie.ups-tlse.fr

${ }^{b}$ Universidade Federal do Vale do Sao Francisco, Av. José de Sá Maniçoba, Petrolina, PE, Brazil

${ }^{c}$ Centre de Microscopie Electronique Appliquée à la Biologie (CMEAB) Faculté de Médecine Rangueil, 133, Route de Narbonne, 31062 Toulouse, France

${ }^{d}$ Institut Clément Ader (ICA), Université de Toulouse, CNRS, IMT Mines Albi, UPS, INSA, ISAE-SUPAERO, Campus Jarlard, CT Cedex 09 81013, Albi, France

$\dagger$ Electronic supplementary information (ESI) available. See DOI: 10.1039/d0sm00128g
}

reduction of systemic side effects. ${ }^{16,17}$ Colloidal carriers are interesting for delivering drug to skin, and various were described including, liposomes, niosomes, transferosomes, ethosomes, nanoemulsions, solid lipid nanoparticles (SLNs), and nanostructured lipid carriers (NLCs). ${ }^{18-21}$ Our organogel particles could be very interesting vehicles for delivering lipophilic drugs to the desired site in the skin, but we sought to reduce their size for optimum permeation. For this reason, we decided to prepare organogel particles in the presence of nanostructured fluid. Considering our elaboration process, there were two options, one starting from a microemulsion, and the other from a nanoemulsion. In a first approach we discarded microemulsions because they could lead to a fast bulk gelation (organogel or hydrogel) rather than gelled nanoparticles. ${ }^{22-24}$ Therefore, we preferred to try to prepare organogel nanoparticles from a nanoemulsion. Nanoemulsions (NanoE) are submicrometer-sized dispersed droplets, in the $20-200 \mathrm{~nm}$ range. ${ }^{25,26}$ Due to their small size, nanoemulsions are transparent or translucent systems that present low viscosity and high stability against sedimentation or creaming. ${ }^{27}$ This stability and a large interfacial area often make nanoemulsions suitable for use as drug carriers for improving the delivery of therapeutic agents. ${ }^{28-30}$ Nanoemulsions can be defined as oil-in-water or water-in-oil, and contain an emulsifier (or a mixture) that is critical for the creation of small-sized droplets. ${ }^{27}$ It is possible to prepare nanoemulsions through various methods that can be classified as high-energy and low-energy preparations. 
Among the low-energy methods, phase inversion temperature (PIT) is the most widely used in the industry, ${ }^{28}$ and was particularly suited to our experiment. PIT emulsification is based on the changes in the solubility (curvature) of surfactants depending on the temperature. ${ }^{33-37}$ For example, nonionic surfactants of the polyoxyethylene kinds are hydrophilic and soluble in water at low temperatures. ${ }^{30}$ When the temperature is raised, they turn lipophilic and become more soluble in the oil phase. Before that, they reach a spontaneous curvature close to zero corresponding to the PIT. From this point, rapid cooling results in the spontaneous formation of small oil droplets due to the polarity changes of the surfactants that migrate from the oil phase into the aqueous phase. For our experiment we needed a nanoemulsion system with a PIT point above the gel temperature of the organogel, as required by our particle preparation process. ${ }^{1-4}$

However, contrary to what we expected, we did not obtain organogel nanoparticles from an oil-in-water nanoemulsion, but surprisingly a new and interesting gelled nanoemulsion that we describe here for the first time. The goal of this study is to analyze and compare the behaviour of the two starting systems (nanoemulsion and organogel) with the gelled nanoemulsion, and to understand the gelation transfer.

\section{Results and discussion}

Preparation and characterization of the starting nanoemulsion

The formation of a PIT system requires determining a precise phase diagram, which is a long and tedious study, so we used an unique mixture inspired by the system described by Sadurní et al. ${ }^{28}$ consisting of Polyoxyl 40 hydrogenated castor oil (Kolliphor ${ }^{\circledR}$ RH40) and lauryl ether (Brij ${ }^{\circledR}$ L4) as surfactants, and a triglyceride medium chain (Miglyol ${ }^{\mathbb{B}} 812$ ) as oil (structures are reported in ESI, $\dagger$ Fig. S1). These ingredients were used in proportions of $0.68 \mathrm{~g}-0.32 \mathrm{~g}-0.5 \mathrm{~g}$, respectively, for $8.5 \mathrm{~g}$ of water, providing a PIT point of $72{ }^{\circ} \mathrm{C}$.

During the PIT phenomenon, the optical appearance of the colloidal dispersion changes from opaque macroemulsion at high temperature to bluish translucent nanoemulsion after cooling to room temperature (visual appearance and observations by optical microscopy are reported in Fig. S2 and S3, ESI $\dagger$ ). This change can be followed by turbidimetric measurements, showing a sharp increase in absorbance at $72{ }^{\circ} \mathrm{C}$ related to the PIT. This temperature transition is fully reversible and reproducible. Complementary measurements were performed by multi-speckle diffusing wave spectroscopy (MS-DWS). This optical technique provides a non-destructive measurement of the sample's microdynamics (related to the viscosity) over time and/or temperature. They showed a micro-dynamic peak due to molecule movements during inversion phenomenon confirming the PIT of $72{ }^{\circ} \mathrm{C}$ (Fig. S4, ESI $\dagger$ ).

Nanoemulsion was first characterized by rheological measurements. Interestingly, the nanoemulsion's storage modulus $\left(G^{\prime}\right)$ was one order of magnitude higher than the loss modulus $\left(G^{\prime \prime}\right)$ (Fig. S5, ESI $\dagger$ ). This could indicate that there is some cohesive part in the nanoemulsion that we can assume to be a strong interaction between the droplets. ${ }^{31-33}$ Further characterization of the nanoemulsion was then achieved by dynamic and static light scattering (DLS, SLS), small angle X-ray scattering (SAXS), cryo-scanning electron microscopy (Cryo-SEM), and transmission electron microscopy (TEM).

Multi-angle DLS measurements (description of data, analysis, and related equations are reported in the ESI $\dagger$ ) were performed using the 3D-cross correlation technique because the highly opalescent samples producing significant multiple scattering. They indicated the presence of colloidal particles with homogeneous size distribution (hydrodynamic radius was $13.8 \mathrm{~nm}$ ), which is compatible with dispersed $\mathrm{o} / \mathrm{w}$ nanodroplets. Upon aging, the size increased gradually up to $25 \mathrm{~nm}$ after few months (Fig. S6, ESI $\dagger$ ), indicating a coalescence of nanodroplets probably due to attractive inter-particle interactions. Upon dilution, the multiple scattering was suppressed when dilution reached 1/10 and below. Moreover, the apparent hydrodynamic radii decreased from $13.8 \mathrm{~nm}$ to $8.6 \mathrm{~nm}$ upon dilution up to $1 / 20$ (Fig. S7, ESI $\dagger$ ). The larger hydrodynamic radius observed at high concentrations (apparent slower motion) could again be related to attractive interparticle interactions. In multi angle static light scattering (MASLS) experiments (Fig. S8, ESI $\dagger$ ), mainly flat scattering curves were obtained at $q>1 \times 10^{-3} \AA^{-1}$ and $I(q)$ was constant in this zone. Indeed, the particles were too small to reach the Guinier regime and to determine both the form factor $P(q)$ and the radius of gyration $R_{\mathrm{g}}$. At lower $q$ values, an increase of the scattered light intensity was observed, which could possibly indicate the presence of a large, fractal-like network of particles as often observed in concentrated nanoemulsions. ${ }^{34,35}$ SAXS measurements were performed on native nanoemulsion and on diluted samples (Fig. S9, ESI $\dagger$ ). Concentrated solutions exhibited a marked correlation peak related to inter-particle interactions. It was observed at $q=0.0196 \AA^{-1}$ (interaction distance of $32.1 \mathrm{~nm}$ ), confirming that the particles are closely distributed. Upon dilution, this peak gradually migrated to lower $q$ values and finally disappeared for $1 / 10$ dilution and below. The $1 / 10$ dilution sample was used to determine the form factor $P(q)$. A core-shell model was well suited to fit the experimental data (radius of the inner oil core $=9.6 \mathrm{~nm}$; thickness of surfactant layer $=1.4 \mathrm{~nm}$ ). For concentrated samples an additional sticky hard sphere $S(q)$ structure factor was used to take into account attractive inter-particle interactions (analytical descriptions of $P(q)$ and $S(q)$ are reported in the $\mathrm{ESI} \dagger)$.

The nanoemulsion structure was then studied by electron microscopies. TEM showed very densely packed, uncoalesced spherical droplets (Fig. 1), with a mean diameter of $26 \pm 2 \mathrm{~nm}$ in accordance with the scattering experiments and confirmed by cryo-SEM (Fig. S10, ESI $\dagger$ ).

\section{Preparation and characterization of the reference organogel}

We prepared a binary organogel with a gelation temperature $T_{\text {gel }}$ compatible with nanoemulsion formation $\left(T_{\text {gel }}<\right.$ PIT $)$.

The oil phase was Miglyol ${ }^{\mathbb{R}} 812$, a biocompatible mediumchain triglyceride used in toxicology studies. ${ }^{36}$ As organogelator we selected 12-hydroxyoctadecanoic acid (12-HOA), also biocompatible and used in pharmaceutical applications. ${ }^{37}$ Another point of 


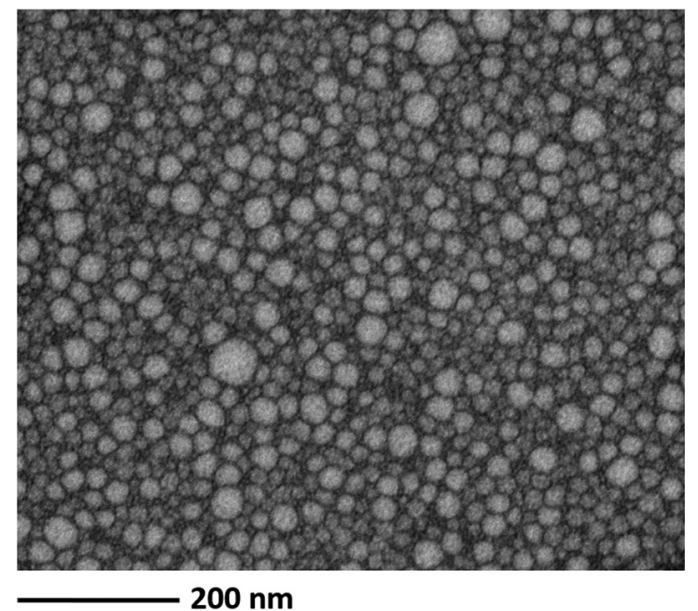

Fig. 1 TEM micrograph of the nanoemulsion after negative staining.

interest of 12-HOA lies in its gelling power, allowing the gelation of many oils and organics solvents, even at very low concentrations. ${ }^{10-15}$

As expected, 12-HOA was able to gellify the Miglyol ${ }^{\mathbb{R}} 812$ between 1-25 wt\% (as illustrated by the inverted bottle test in Fig. S11, ESI $\dagger)$. Gelation and melting temperatures $\left(T_{\text {gel }}\right.$ and $\left.T_{\text {melt }}\right)$ were determined by differential scanning calorimetry (DSC) at various concentrations (Fig. S12, ESI $\dagger$ ). All compositions tested had gelation and melting temperatures compatible with the nanoemulsion PIT. In view of further including 12-HOA in the nanoemulsion droplets, which could notably present stability for drug delivery applications, ${ }^{4}$ we fixed the composition at $20 \mathrm{wt} \%$ $\left(T_{\text {gel }}=56{ }^{\circ} \mathrm{C}\right)$. 12-HOA's organization in Miglyol ${ }^{\circledR} 812$ was characterized by optical microscopy, SAXS analysis, and TEM.

Micrographs of $20 \mathrm{wt} \% 12-\mathrm{HOA}$ in Miglyol $^{\circledR} 812$ under cooling are reported in ESI $\dagger$ (Fig. S13). Above $T_{\text {gel }}, 12$-HOA is fully soluble resulting in a homogeneous and transparent solution. With cooling, rapid gel formation is observed at $61{ }^{\circ} \mathrm{C}$ : long, increasingly dense fibers are gradually formed up to $25{ }^{\circ} \mathrm{C}$. The SAXS characterization of the 12-HOA organogel in Miglyol $^{\circledR} 812$ (Fig. S14, ESI $\dagger$ ) is very similar to what was observed by others in organogels formed by 12 -HOA in various organic solvents. ${ }^{38-40}$ Indeed, 12-HOA gelation usually results from a self-assembled fibrillar network (SAFIN). ${ }^{41,42}$ The SAXS data were adjusted according to Laupheimer et al., ${ }^{40}$ using a model combining stiff cylinders representing the gelator fibers and lamellae representing their lamellar junction nodes (an analytical description is reported in the ESI $\dagger$ ), confirming the formation of an interconnected fibrillary network. In this fit, the radius of the infinitely long cylinders was found to be $r=10.6 \mathrm{~nm}$ and the lamellae presented a thickness of $T=$ $33.3 \mathrm{~nm}$, these values being very close to what was observed for 12-HOA gels in decane. ${ }^{40}$ In addition, the presence of a Bragg peak at $q=0.132 \AA^{-1}$ (distance $\left.=4.8 \mathrm{~nm}\right)$ indicated a partial crystalline character of the fibers and the nodes. ${ }^{38,39}$ TEM observations (Fig. 2) revealed that the organogel in Miglyol $^{\circledR}$ 812 can adopt twisted ribbon structures with high length-towidth ratios. The lengths of the ribbons can reach several hundred micrometers, and they have a left-handed twist.

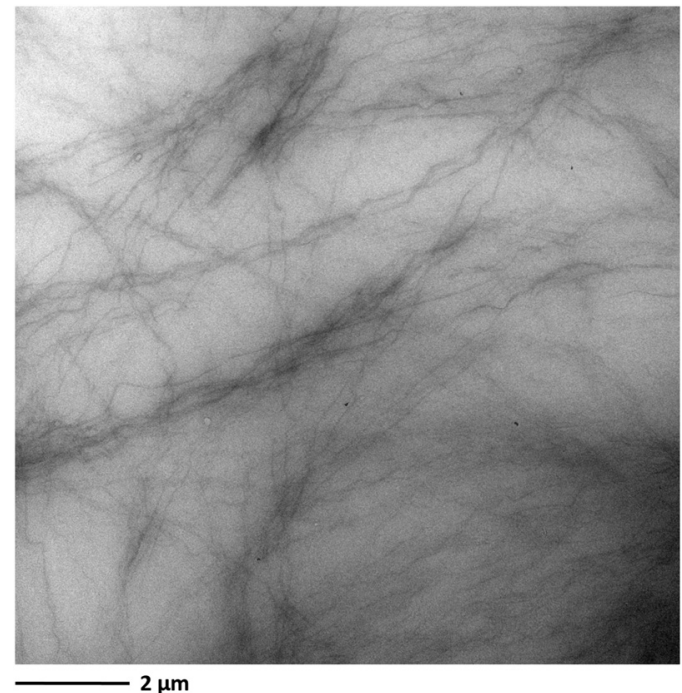

Fig. 2 TEM micrograph of the organogel after negative staining.

\section{Gelation of the nanoemulsion}

The coexistence of different self-assembled structures formed inside the same system is not unique, and many examples exist in nature or have already been formulated. ${ }^{43-50}$ Gelled microemulsions have been described, leading to the coexistence of a water-in-oil microemulsion with a gelatin network, ${ }^{22,23}$ or a bicontinuous microemulsion with a self-assembly of 12-HOA. ${ }^{24}$ The latter may be considered as a particular organogel in which the gelled solvent is the bicontinuous phase of the microemulsion. There is currently no example of the coexistence of an oil-in-water nanoemulsion with a self-assembled network of gel, the two resulting from the organization of a unique system.

\section{Hydrogel formation and gelation process}

After cooling the nanoemulsion containing the 12-HOA prepared using the PIT process, we observed the formation of a gel confirmed by the inverted bottle test (Fig. 3). Above the PIT, the system appears white and opaque, exactly like the system without 12-HOA. Decreasing the temperature below the PIT led to a fairly transparent bluish solution characteristic of the formation of nanometric droplets. But the opacity of the solution increased rapidly. This increase of opacity is associated with an increase of viscosity. After a few days we can observe the formation of an opaque gel.

The gelation process related to the self-assembly of the 12-HOA in the external phase was relatively slow. Gelation kinetics was

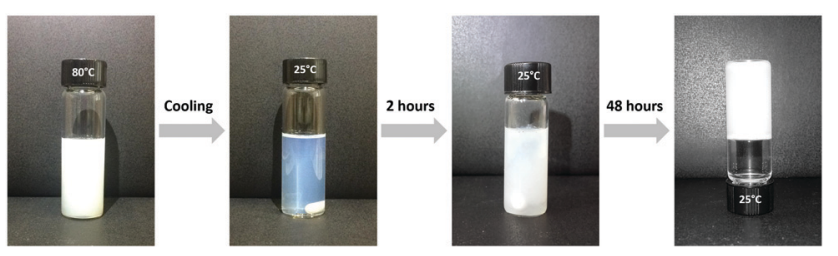

Fig. 3 Photographs showing the hydrogel formation with cooling and aging. 

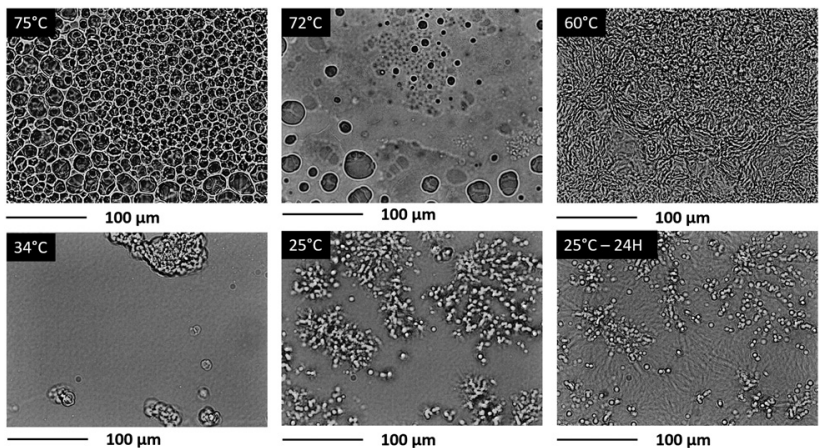

Fig. 4 Optical micrographs of hydrogel formation with cooling and aging.

followed by MS-DWS (Fig. S15, ESI $\dagger$ ). A first rapid decrease of the micro-dynamics is due to cooling and the formation of a weak gel in the first hours. Then a slower gelation occurs with aging, the gel becoming stable from 3 days (the gel nature was confirmed by rheological measurements, Fig. S16, ESI $\dagger$ ). The first part of this gelation process has been observed by optical microscopy under cooling, showing different steps (Fig. 4). We observed a transition at $72{ }^{\circ} \mathrm{C}$ (corresponding to the PIT) from a dense emulsion to a more transparent system with a lower density of large droplets. From $60{ }^{\circ} \mathrm{C}$ to $34{ }^{\circ} \mathrm{C}$ we observed a new type of transient organization that progressively converts into dispersed small aggregates, leading finally to the apparition of a fibrillary texture at $25{ }^{\circ} \mathrm{C}$, which then slowly expanded upon aging.

Further characterization was performed using turbidimetric measurements. Again a multi-step profile was obtained under cooling. Contrary to the nanoemulsion, this profile was modified when subjecting the gel to heating (Fig. 5).

The peaks at higher temperatures correspond to the PIT of the system and are $73.5{ }^{\circ} \mathrm{C}$ and $70.5{ }^{\circ} \mathrm{C}$ for the cooling and heating stages, respectively. Considering the cooling sequence, we first observed a rapid decrease in absorbance (region A) until $66{ }^{\circ} \mathrm{C}$, corresponding to the formation of the nanoemulsion below the PIT. From $66{ }^{\circ} \mathrm{C}$ to $54{ }^{\circ} \mathrm{C}$ (region B), we observed a rapid increase in absorbance then a stabilization (starting of self-assemblies in the droplets). Below $54{ }^{\circ} \mathrm{C}$ (region $\mathrm{C}$ ) the absorbance slowly decreased until $32{ }^{\circ} \mathrm{C}$ (exit of the first assembles outside the droplets). Finally, we observed a rapid and continuous increase in absorbance corresponding to the gel maturation (growing of ribbons and fibers in the aqueous phase). Below $54{ }^{\circ} \mathrm{C}$ the absorbance slowly decreased until $32{ }^{\circ} \mathrm{C}$ (region $\mathrm{C}$ ). Finally, we observed a rapid and continuous increase in absorbance corresponding to the gel maturation. Regarding the heating sequence, we also found four regions, but with a different profile and small shifts in temperatures. From $25{ }^{\circ} \mathrm{C}$ to $44^{\circ}$ we observed a decrease in absorbance to a very low value (region A). The sample's transparency was maintained until $52{ }^{\circ} \mathrm{C}$ (region B). We then observed a slow increase in absorbance until $66{ }^{\circ} \mathrm{C}$ (region $\mathrm{C}$ ), followed by a rapid, major increase at the PIT (region D). A close behaviour was obtained by rheological measurements showing four successive steps occurring at similar temperatures (Fig. S16, ESI $\dagger$ ).

These complementary experiments have revealed that the gelation process has a thermal profile which is much more complex than what observed for each parent system (nanoemulsion and organogel). It is also different from their simple combination, suggesting a non-orthogonal system contrary to what has been reported in the case of gelled microemulsions. ${ }^{40}$

\section{Characterization of the hydrogel structure}

Hydrogel SAXS characterization globally showed characteristic features of both a 12-HOA organogel and a nanoemulsion, as presented in Fig. 6. However, the signal from the gelled nanoemulsion was not the simple addition of the signals from the
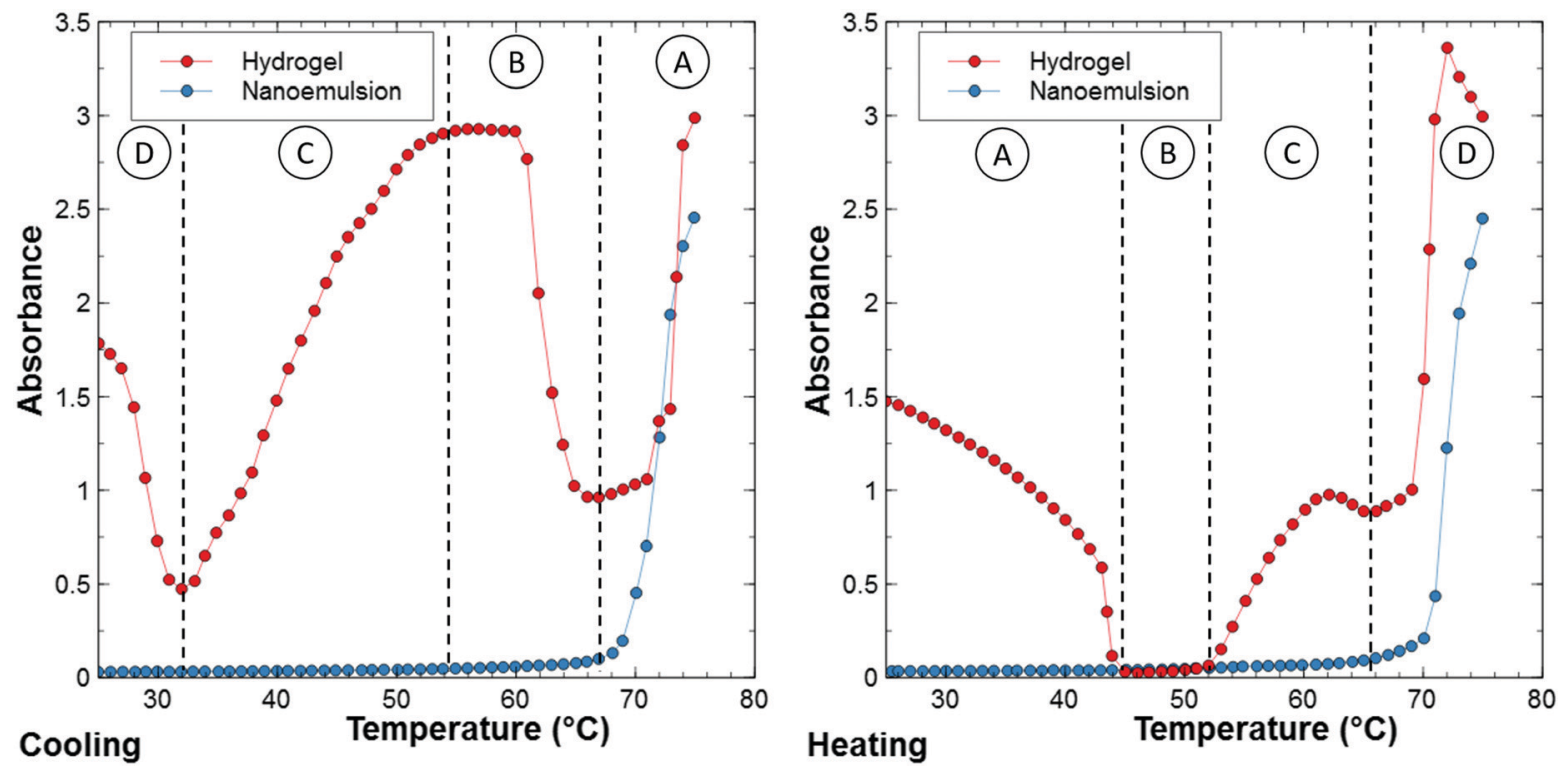

Fig. 5 Absorbance recorded at $500 \mathrm{~nm}$ of the native nanoemulsion (blue curves) and the final hydrogel (red curves) as a function of the temperature under cooling (left) and heating (right). 


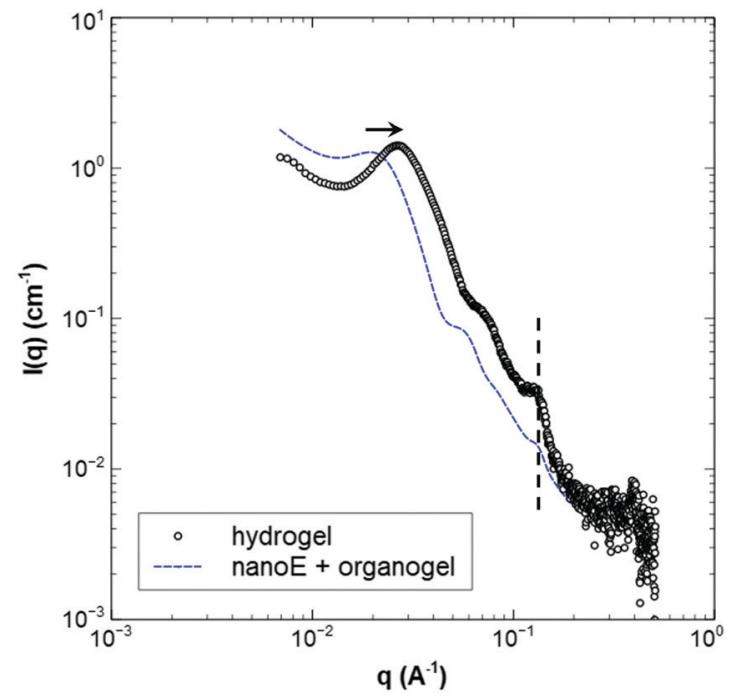

Fig. 6 SAXS profile of the gelled nanoemulsion (open circles) and sum of the fitted SAXS profiles of 12-HOA organogel and native nanoemulsion (doted blue line) shown for comparison. The vertical doted black line indicates the position of the Bragg peak, while the arrow indicates the shift of the interaction peak.

two parent constituents, confirming the non-orthogonality of the system.

The Bragg peak located at $q=0.132 \AA^{-1}$ (corresponding to the crystalline character of the fibrillary network) did not shift, showing that the gelation properties of 12-HOA were not much affected by the presence of the nanoemulsion and the aqueous environment. This supports the idea that the gelation process occurs first in the oil phase inside the nanoemulsion droplets. In contrast, the contribution of the nanoemulsion at low $q$ values was modified under SAXS, especially with a more pronounced interaction peak that also shifted from $1.96 \times$ $10^{-2}$ to $2.65 \times 10^{-2} \AA^{-1}$. This indicated that the droplets were in stronger interaction and at a shorter interacting distance $(23.7 \mathrm{~nm})$ than in the native nanoemulsion. This much tighter packing could facilitate the growth of 12-HOA fibers and the connection of neighboring droplets.

The characterization was completed by electron microscopy observations on stabilized samples. Cryo-SEM preserved fine nanoscale details and showed long, twisted ribbons of 12-HOA ( $>1 \mu \mathrm{m}$ length), mixed with a dense population of nanoemulsion droplets (mean diameter $=20 \mathrm{~nm}$ ) as shown in Fig. 7 (left).

The droplets look similar to those in the native nanoemulsion in terms of their size and morphology. This confirms their preservation, even after the gelation transfer of the 12-HOA from the oil phase to the water phase. For a better observation of fiber morphology, we performed TEM microscopy on a diluted sample. Fig. 7 (right) shows long $(>100 \mu \mathrm{m})$ and wide (10-100 nm) left-hand twisted ribbons of 12-HOA. This morphology is of the same kind as that observed in the gelation of the oil phase (Fig. 2), and suggests the same supramolecular organization of 12-HOA in water. This later observation was surprising, as 12-HOA had never yet been described as a hydrogelator.
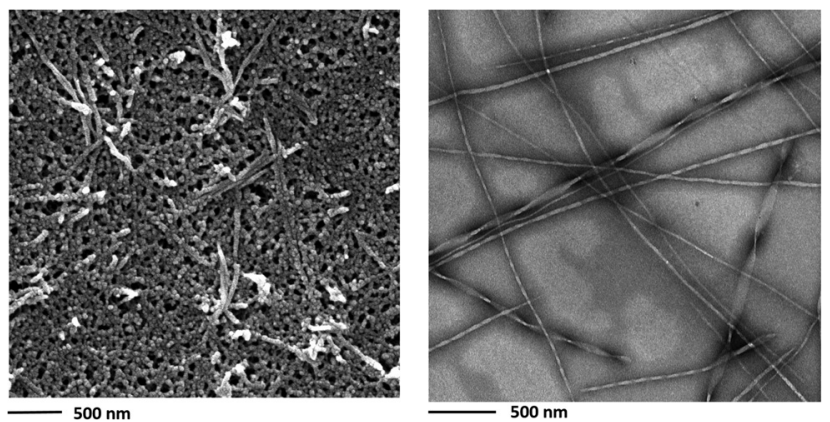

Fig. 7 (left) Cryo-SEM micrograph of the hydrogel. (right) TEM micrograph of the hydrogel after negative staining.

\section{Suggested gelation mechanism}

In order to explain the formation of the gelled nanoemulsion, we propose the hypothesis illustrated in Fig. 8.

In a first step (Fig. 8, step a), in a temperature range between the $T_{\text {gel }}$ of the 12-HOA in the oil phase and the PIT temperature $\left(55{ }^{\circ} \mathrm{C}<T^{\circ}<72{ }^{\circ} \mathrm{C}\right.$ ), the organogelator is totally soluble inside the droplets of the nanoemulsion. Beyond the $T_{\text {gel }}$, the 12-HOA starts to self-assemble inside the droplets (Fig. 8, step b). Under gelating conditions, 12-HOA starts to forms elementary bricks of self-assembled molecules that lead to pieces of ribbons, fibrils, and finally a three-dimensional network of fibers with lateral dimensions on the submicron scale. ${ }^{51,52} 12$-HOA cannot form a hydrogel directly because it is insoluble in water, even at high temperatures. Moreover, assembly is also based on hydrogen bonds that would be highly disturbed in water. Here we observed that the gelation starts inside the oil phase of the nanodroplets and continues in the water phase. This is an interesting example of self-assembling starting in an oil phase and growing in a water phase. Recently K. Aramaki et al. have also described an original transfer of gelation after cooling, but in this case from a micellar phase with 12-HOA dissolved in its melted state. ${ }^{53}$ In our case, this phenomenon can be explained by the structure formed by the elementary brick of 12-HOA selfassembling, as described in the literature in apolar solvents. ${ }^{54}$ As illustrated in Fig. 8, six-membered ringlike bundles of 12-HOA molecules are connected head-to-head through a mechanism similar to acetic acid dimerization. ${ }^{55}$ This elementary brick can self-assemble and grow by van der Waals interactions inside the nanodroplets, leading to very small pieces of ribbon. This step corresponds to the $\mathrm{B}$ region of the cooling curve of absorbance (Fig. 5), with an increase in turbidity because of the organization of 12-HOA inside the droplets. The limited volume of the oil phase inside the nanodroplets cannot contain the growth of the 12-HOA self-assembly. Consequently, as the temperature decreases we have an exchange of material favoured by the fact that the nanodroplets become closer, with possible connections leading to the superassembly of droplets (Fig. 8, step c). This last assembly could correspond to the structures observed using optical microscopy at $60{ }^{\circ} \mathrm{C}$ (Fig. 4). This step corresponds to the $\mathrm{C}$ region of the cooling curve of absorbance (Fig. 5). In this region, turbidity decreases because the initial 12-HOA assemblies exit the droplets, diluting and growing as the temperature decreases. At room temperature, 
(a)
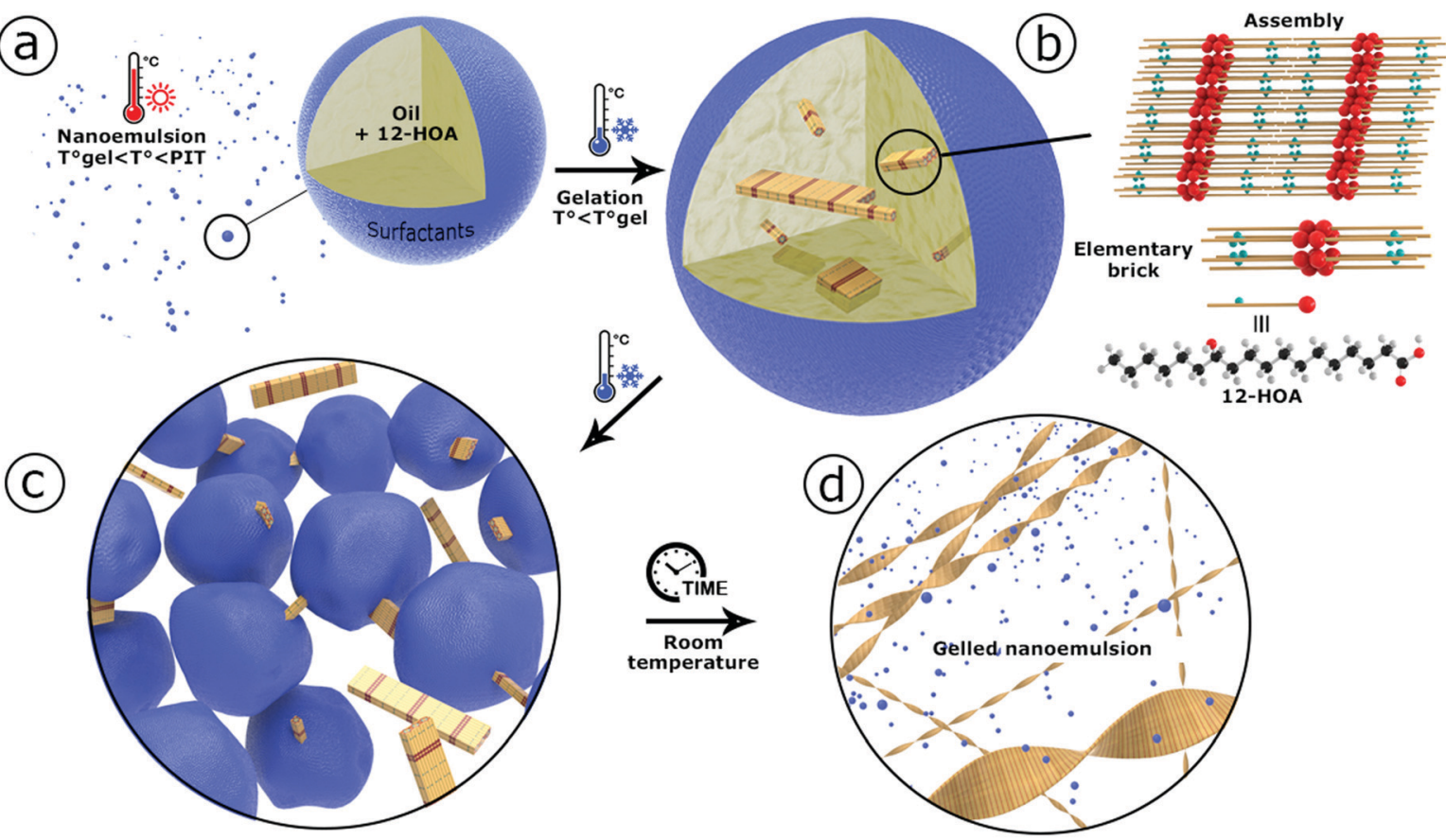

Fig. 8 Different steps suggested for the gelation mechanism.

they slowly form large, twisted ribbons that progressively selfassemble into fibers dispersed in the nanoemulsion (Fig. 8, step d). The stability of the assembly in water could be also improved by adsorption of free surfactant at the surface of the ribbons, and by nanoemulsion droplets as we observed by electron microscopy. This last step corresponds to the $\mathrm{D}$ region of the cooling curve of absorbance (Fig. 5), with a slow increase in absorbance corresponding to the formation of the $3 \mathrm{D}$ network of fibers with a growing number of junction zones, increasing the opacity of the hydrogel.

\section{Experimental}

\section{Materials}

Polyoxyl-40 hydrogenated castor oil (Kolliphor ${ }^{\circledR}$ RH 40) and polyoxyethylene (4) lauryl ether $\left(\mathrm{Brij}^{\circledR} \mathrm{L} 4\right)$ were purchased from Sigma-Aldrich (France). The triglyceride medium-chain Miglyol ${ }^{\mathbb{R}}$ 812 was a gift from Robertet (France). 12-Hydroxyoctadecanoic acid (12-HOA, flakes, technical grade) was purchased from Alberdingk Boley (Germany). All products were used as received. Chemical structures are reported in the ESI $\dagger$ (Fig. S1). The water used in all experiments was produced by a two-stage Milli-Q filtration system from Millipore and had a resistivity higher than $18.2 \mathrm{M} \Omega \mathrm{cm}$.

\section{Gelled nanoemulsion preparation}

The gelled nanoemulsion was prepared using the phase inversion temperature (PIT) method. Kolliphor $\left.{ }^{(}\right)$RH $40(0.68 \mathrm{~g})$ and Brij ${ }^{\circledR}$ L4 (0.32 g) surfactants were mixed with the Miglyol ${ }^{\circledR} 812$ (0.4 g) oil and 12-HOA $(0.1 \mathrm{~g})$ gelator in a glass vial closed by a septum and stirred at $80{ }^{\circ} \mathrm{C}$ in a water bath for $10 \mathrm{~min}$ to ensure homogeneity of the mixture. In parallel, water $(8.5 \mathrm{~g})$ was also heated to $80{ }^{\circ} \mathrm{C}$ and progressively added to the organic mixture under constant stirring. The resulting dispersion was then removed from the bath and left to cool at room temperature.

\section{Optical microscopy}

Optical observations were performed at various temperatures on an Olympus BX53 microscope with a heating/cooling temperature controller (Mettler Toledo FP82HT) at the sample stage. The temperature was adjusted with an accuracy of $0.1{ }^{\circ} \mathrm{C}$. The transitions were monitored by a microscope video system. The images from the microscope were converted to digital images through a color video camera (Olympus SC30). A series of images was obtained during the process and analyzed using Stream Basic image processing software from Olympus, version 1.9.1. Polarized optical microscopy can also be performed with the same microscope using an additional polarizer. The samples were prepared between slide and cover glass sealed with varnish to avoid evaporation.

\section{Turbidimetric measurements}

A Cary 100 Bio UV-visible spectrophotometer from Varian was used to measure the turbidity of the dispersions upon heating and cooling. Absorbance at $500 \mathrm{~nm}$ was measured using $2 \mathrm{~mL}$ of sample in a closed quartz cell $(10 \times 10 \mathrm{~mm})$ from Hellma. The temperature was increased from 25 to $75{ }^{\circ} \mathrm{C}$ at a rate of $5{ }^{\circ} \mathrm{C} \mathrm{min}{ }^{-1}$ to avoid phase separation at high temperature. Then the temperature was decreased to $25{ }^{\circ} \mathrm{C}$ at the same rate. Another heating/cooling cycle was performed to check the reversibility of the system. Water was used as a blank for the nanoemulsion and the gelled nanoemulsion.

\section{SAXS}

SAXS measurements were conducted on a Xeus 2.0 SAXS beamline for laboratory from Xenocs (FERMAT Federation, Toulouse), with an internal copper source producing $8 \mathrm{keV}$ X-rays (wavelength $=1.54 \AA$ ). 
Signals were recorded using a Pilatus $1 \mathrm{M}$ pixel detector from Dectris. The sample-to-detector distance was $1216.5 \mathrm{~mm}$ so that the $q$ range covered was $6 \times 10^{-3}$ to $5 \times 10^{-1} \AA^{-1}$. For each sample, 6 scans of $20 \mathrm{~min}$ were recorded and averaged. The observed intensities $I(q)$ were corrected for transmission, solvent, and detector response. Absolute intensities $I(q)\left(\mathrm{cm}^{-1}\right)$ were obtained after standard normalization. Analyses of the $I(q)$ profiles were performed using SasView software (http://www.sasview.org/), version 4.2.2.

\section{Transmission electron microscopy (TEM)}

The organogel, nanoemulsion and gelled nanoemulsion were studied by TEM. For this study, 20-microliter drop of samples solution were deposited on a Collodion/carbon coated copper grids. After one minute, the grids were then stained for 10 seconds by inversion onto a drop of $2 \%$ uranyl aqueous solution. The grids were blotted using filter paper after the staining step. The nanoemulsion and gelled nanoemulsion have been diluted $(\times 20)$ for observations. The organogel samples were deposited on the grid at $T=80{ }^{\circ} \mathrm{C}$. Observations were performed at $80 \mathrm{kV}$ with an Hitachi HT7700 transmission electron microscope.

\section{Cryo-scanning electron microscopy (Cryo-SEM) after high-pressure freezing (HPF)}

Cryo-SEM was used to observe the nanoemulsion and the gelled nanoemulsion. For this technique, the samples were first inserted between two HPF specimen carriers dedicated to cryofracture (Leica) and immediately loaded into a HPM100 HPF unit (Leica). The sample were fixed and frozen within $5 \mathrm{~ms}$ at 2050 bar and samples were then transferred to cryovials under liquid nitrogen. Then the HPF specimen carrier with the freezing solution was inserted into the Quorum PP3000T preparation chamber. For insertion, specimen cryo shuttles and stubs with the right HPF specimen carrier adapter were used in accordance with the description in the publication by Payre B. and coll. ${ }^{56}$ After quick transfer under vacuum into the preparation chamber, the samples were fractured at $-140{ }^{\circ} \mathrm{C}$, sublimed at $-95{ }^{\circ} \mathrm{C}$ for $30 \mathrm{~min}$ and then coated using platinum sputtering. Lastly, they were transferred to the cryo-SEM Quanta 250 FEG chamber and kept at $-140{ }^{\circ} \mathrm{C}$ for observation with an FEI Quanta 250 FEG scanning electron microscope at an accelerating voltage of $5 \mathrm{kV}$.

\section{Conclusions}

This work demonstrated an original example of organogelator self-assembly growing from an oil phase to a water phase until a hydrogel was formed. Surprisingly, we observed the coexistence of an oil-in-water nanoemulsion with a self-assembled network of gel, the two resulting from the organization of a unique system. We observed and characterized the system using various techniques (turbidimetry, rheology and optical microscopy), demonstrating the totally modified thermal behavior of the nanoemulsion under both cooling and heating. We observed successive steps indicating different organizations of the system. Based on these results and on electron microscopy observations, we proposed a gelation mechanism. When the temperature reaches the $T_{\text {gel }}$ when cooling, there is a first step corresponding to an initial assembly of 12-HOA inside the oil nanodroplets. As the assemblies grow larger, they leave the droplets and exchange as long as the droplets interact strongly. As the temperature decreases, twisted ribbons are progressively formed in water, slowly leading to a 3D network of entangled fibers and finally a stable hydrogel after 3 days of equilibration at room temperature. This growth in the water phase was made possible because the elementary brick of 12-HOA was formed in the oil phase, ${ }^{45}$ and because its subsequent assemblies were not disturbed in the presence of water.

Our initial goal was to gel droplets oil in a nanoemulsion and to elaborate organogel nanoparticles to enhance skin permeation by the active molecules. Instead, we obtained a new system corresponding to a nanoemulsion entrapped in a hydrogel formed by the three-dimensional self-assembly of the organogelator. This original system is just as interesting, because we keep the interest of the nanoemulsion (size and stability), associated with a gel for improved controlled release of the encapsulated substances. Moreover, the gel to sol transition of our system is also tunable by temperature, simply by adjusting the 12-HOA concentration. Recently, thermoresponsive nanoemulsion-based gels have also been described, demonstrating the interest of such systems for skin delivery. ${ }^{57}$ These new systems could also find applications in cosmetics with new textures, or in the field of cleansing by combining nanoemulsion (ultra low surface tension) and gel (high viscosity).

\section{Conflicts of interest}

The authors report no conflicts of interest. The authors alone are responsible for the content and writing of the paper.

\section{Acknowledgements}

We gratefully thank the CMEAB-Toulouse for the Cryo SEM images. (Faculté de Médecine de Rangueil-Université Toulouse III). This work benefited from the use of the SasView application, originally developed under NSF award DMR-0520547. SasView contains code developed with funding from the European Union's Horizon 2020 research and innovation program under the SINE2020 project, grant agreement No 654000. The research federation FERMAT (Université de Toulouse, France) is acknowledged for providing access to the Xeuss 2.0 SAXS instrument (Xenocs) through the CPER I-MATECBIO 2015-2020 grant funded by the Région Occitanie and Toulouse Métropole, and Dr P. Roblin from the Laboratoire de Génie Chimique de Toulouse is thanked for technical support in SAXS measurements. We acknowledge the EU for financial support (FEDER-35477: Nano-objets pour la biotechnologie).

\section{Notes and references}

1 P. Kirilov, L. Lukyanova, S. Franceschi-Messant, V. Perier, E. Perez and I. Rico-Lattes, Colloids Surf., A, 2008, 328, 1-7.

2 A. Boudier, P. Kirilov, S. Franceschi-Messant, H. Belkhelfa, L. Hadioui, C. Roques, E. Perez and I. Rico-Lattes, J. Microencapsulation, 2010, 27, 682-692. 
3 M. P. Siqueira-Moura, S. Franceschi-Messant, M. Blanzat, M. I. Ré, E. Perez, I. Rico-Lattes, A. Lattes and A. C. Tedesco, J. Colloid Interface Sci., 2013, 401, 155-160.

4 B. Martin, F. Brouillet, S. Franceschi and E. Perez, AAPS PharmSciTech, 2017, 18, 1261-1269.

5 H. Ihara, T. Yamada, M. Nishihara, T. Sakurai, M. Takafuji, H. Hachisako and T. Sagawa, J. Mol. Liq., 2004, 111, 73-76.

6 X. Luo, W. Xiao, Z. Li, Q. Wang and J. Zhong, J. Colloid Interface Sci., 2009, 329, 372-375.

7 K. Iwanaga, M. Kawai, M. Miyazaki and M. Kakemi, Int. J. Pharm., 2012, 436, 869-872.

8 F. R. Lupi, V. Greco, N. Baldino, B. de Cindio, P. Fischer and D. Gabriele, J. Colloid Interface Sci., 2016, 483, 154-164.

9 M. A. Cerqueira, L. H. Fasolin, C. S. F. Picone, L. M. Pastrana, R. L. Cunha and A. A. Vicente, Food Res. Int., 2017, 96, 161-170.

10 T. Tachibana and H. Kambara, J. Colloid Interface Sci., 1968, 28, 173-174.

11 M. Burkhardt, S. Kinzel and M. Gradzielski, J. Colloid Interface Sci., 2009, 331, 514-521.

12 V. A. Mallia and R. G. Weiss, J. Phys. Org. Chem., 2014, 27, 310-315.

13 C. Liu, M. Corradini and M. A. Rogers, Colloid Polym. Sci., 2015, 293, 975-983.

14 M. Burkhardt, L. Noirez and M. Gradzielski, J. Colloid Interface Sci., 2016, 466, 369-376.

15 M. A. Rogers, A. J. Wright and A. G. Marangoni, Curr. Opin. Colloid Interface Sci., 2009, 14, 33-42.

16 S. C. McNeill, R. O. Potts and M. L. Francoeur, Pharm. Res., 1992, 9, 1422-1427.

17 W. Leppert, M. Malec-Milewska, R. Zajaczkowska and J. Wordliczek, Molecules, 2018, 23, 681.

18 H. Schreier and J. Bouwstra, J. Controlled Release, 1994, 30, 1-15.

19 G. Cevc and G. Blume, Biochim. Biophys. Acta, Biomembr., 2001, 1514, 191-205.

20 S. Baboota, F. Shakeel, A. Ahuja, J. Ali and S. Shafiq, Acta Pharm., 2007, 57, 315-332.

21 E. Souto, S. Wissing, C. Barbosa and R. Müller, Int. J. Pharm., 2004, 278, 71-77.

22 P. J. Atkinson, M. J. Grimson, R. K. Heenan, A. M. Howe and B. H. Robinson, J. Chem. Soc., Chem. Commun., 1989, 1807.

23 P. J. Atkinson, B. H. Robinson, A. M. Howe and R. K. Heenan, J. Chem. Soc., Faraday Trans., 1991, 87, 3389.

24 M. Laupheimer, K. Jovic, F. E. Antunes, M. da Graça Martins Miguel and C. Stubenrauch, Soft Matter, 2013, 9, 3661.

25 C. Sol, P. Izquierdo, J. Nolla, N. Azemar and M. Garci, Curr. Opin. Colloid Interface Sci., 2005, 10, 102-110.

26 A. Gupta, H. B. Eral, T. A. Hatton and P. S. Doyle, Soft Matter, 2016, 12, 2826-2841.

27 N. Anton, J.-P. Benoit and P. Saulnier, J. Controlled Release, 2008, 128, 185-199.

28 N. Sadurní, C. Solans, N. Azemar and M. J. García-Celma, Eur. J. Pharm. Sci., 2005, 26, 438-445.

29 M. Kumar, A. Misra, A. K. Babbar, A. K. Mishra, P. Mishra and K. Pathak, Int. J. Pharm., 2008, 358, 285-291.

30 A. Kaur, S. S. Katiyar, V. Kushwah and S. Jain, Nanomedicine, 2017, 13, 1473-1482.
31 V. V. Erramreddy and S. Ghosh, Colloids Surf., A, 2015, 484, 144-152.

32 V. V. Erramreddy, S. Tu and S. Ghosh, RSC Adv., 2017, 7, 47818-47832.

33 A. Patel, N. Longmore, A. Mohanan and S. Ghosh, ACS Omega, 2019, 4, 11791-11800.

34 J. N. Wilking, S. M. Graves, C. B. Chang, K. Meleson, M. Y. Lin and T. G. Mason, Phys. Rev. Lett., 2006, 96, 015501.

35 M. E. Helgeson, Y. Gao, S. E. Moran, J. Lee, M. Godfrin, A. Tripathi, A. Bose and P. S. Doyle, Soft Matter, 2014, 10, 3122-3133.

36 N. Buss, P. Ryan, T. Baughman, D. Roy, C. Patterson, C. Gordon and R. Dixit, J. Appl. Toxicol., 2018, 38, 1293-1301.

37 S. R. Pereira Camelo, S. Franceschi, E. Perez, S. Girod Fullana and M. I. Ré, Drug Dev. Ind. Pharm., 2016, 42, 985-997.

38 P. Terech, V. Rodriguez, J. D. Barnes and G. B. McKenna, Langmuir, 1994, 10, 3406-3418.

39 P. Terech, D. Pasquier, V. Bordas and C. Rossat, Langmuir, 2000, 16, 4485-4494.

40 M. Laupheimer, T. Sottmann, R. Schweins and C. Stubenrauch, Soft Matter, 2014, 10, 8744-8757.

41 P. Terech and R. G. Weiss, Chem. Rev., 1997, 97, 3133-3160. 42 M. Laupheimer, N. Preisig and C. Stubenrauch, Colloids Surf., A, 2015, 469, 315-325.

43 A. Brizard, M. Stuart, K. van Bommel, A. Friggeri, M. de Jong and J. van Esch, Angew. Chem., Int. Ed., 2008, 47, 2063-2066.

44 A. M. Brizard, M. C. A. Stuart and J. H. van Esch, Faraday Discuss., 2009, 143, 345.

45 S. Dieterich, T. Sottmann and F. Giesselmann, Langmuir, 2019, 35, 16793-16802.

46 K. Steck, N. Preisig and C. Stubenrauch, Langmuir, 2019, 35, 17142-17149.

47 K. Steck, J. H. van Esch, D. K. Smith and C. Stubenrauch, Soft Matter, 2019, 15, 3111-3121.

48 K. Steck, C. Schmidt and C. Stubenrauch, Gels, 2018, 4, 78.

49 S. Koitani, S. Dieterich, N. Preisig, K. Aramaki and

C. Stubenrauch, Langmuir, 2017, 33, 12171-12179.

50 C. Stubenrauch and F. Gießelmann, Angew. Chem., Int. Ed., 2016, 55, 3268-3275.

51 T. Tachibana and H. Kambara, J. Am. Chem. Soc., 1965, 87, 3015-3016.

52 T. Tachibana, S. Kitazawa and H. Takeno, Bull. Chem. Soc. Jpn., 1970, 43, 2418-2421.

53 K. Aramaki, S. Koitani, E. Takimoto, M. Kondo and C. Stubenrauch, Soft Matter, 2019, 15, 8896-8904.

54 R. Gordon, S. T. Stober and C. F. Abrams, J. Phys. Chem. B, 2017, 121, 9223-9233.

55 R. E. Jones and D. H. Templeton, Acta Crystallogr., 1958, 11, 484-487.

56 B. Payre, E. Gontier, A. Jarray, Y. Martinez, J. P. Laugier, A. Delalleau, B. M. Gaillard, I. Anselme, D. Goudounèche, I. Fourquaux, M. Hemati, V. Gerbaud, M. B. Delisle and C. Guilbeau-Frugier, J. Microsc., 2018, 271, 255-265.

57 S. M. Hashemnejad, A. Z. M. Badruddoza, B. Zarket, C. Ricardo Castaneda and P. S. Doyle, Nat. Commun., 2019, 10, 2749. 